Aim(s)/objectives Our aim was to investigate the factors associated with recurrent bacterial STIs in MSM in Brighton, focusing specifically on drug and alcohol use.

Methods We reviewed MSM presenting to our service between September 2014-September 2015 who had had 3 or more repeat attendances with a bacterial STI. We included infectious Syphilis, pharyngeal, rectal and urethral Chlamydia and Gonorrhoea. We collected data on alcohol and recreational drug use.

Results An estimated 11,000 MSM attended during the study period. Of these, 46 MSM had 3 or more bacterial STIs. The median age was 34.5 years 21-57). 26/46 (57\%) were HIV positive. $32 / 46(70 \%)$ had 3 STIs; $10 / 46(22 \%)$ had 4 STIs, 3/46 $(7 \%)$ had 5 STIs and $1 / 46(2 \%)$ had 6 STIs. $14 / 46(30 \%)$ reported hazardous drinking, 31/46 (67\%) reported use of party drugs (including Mephedrone, Crystal Meth, Ecstasy and GHB) and $7 / 46(15 \%)$ reported 'slamming'.

Discussion/conclusion MSM attending multiple times with recurrent bacterial STIs also report high use of alcohol and recreational drug use including slamming. Public health interventions to reduce incidence of STIs should include focusing on drug and alcohol use in MSM.

\section{P053 HIV SCREENING IN THE HIV NEGATIVE POPULATION - A REGIONAL HIV NETWORK AUDIT OF SCREENING OFFER, UPTAKE AND TURN-AROUND TIMES}

${ }^{1}$ Bridie Howe* ${ }^{2}$ Eleanor Lear, ${ }^{3}$ Daisy Ogbonmwan, ${ }^{4}$ Conrad White, ${ }^{1}$ Nathan Sankar, ${ }^{5}$ Babiker Elawad, ${ }^{4}$ Sarah Duncan, ${ }^{6}$ Medhat Basta, ${ }^{4}$ Alison Wardropper, ${ }^{7}$ Sarup Tayal, ${ }^{3}$ Jane Hussey. ${ }^{1}$ Newcastle upon Tyne Hospitals, Newcastle upon Tyne, UK; ${ }^{2}$ Newcastle University, Newcastle upon Tyne, UK; ${ }^{3}$ City Hospitals Sunderland, Sunderland, UK; ${ }^{4}$ County Durham and Darlington NHS Foundation Trust, Durham, UK; ${ }^{5}$ North Tyneside Community Health, Newcastle upon Tyne, UK; ${ }^{6}$ South Tyneside NHS Foundation Trust, Gateshead, UK; ${ }^{7}$ Virgin Care Sexual Health Teeside, Stockton, UK

10.1136/sextrans-2016-052718.107

Background New BHIVA Standards of care for people living with HIV were published in 2013 for proportion of people newly attending sexual health services offered an HIV test in and time from HIV testing to lab reporting and sharing result with patient

Aims Baseline regional audit to assess HIV screening offer, uptake and turn-around times within sexual health services to feedback to commissioners.

Method Standards set from the 'Management of Sexually transmitted Infections' MEDFASH 2014. Retrospective audit of first 30 attendances between 01/09/14 and 30/11/14. Services reviewed notes coded as either HIV testing performed, inappropriate or declined. Information collected included documentation of offer, reason given for decline or deemed inappropriate. For those tested, times taken for lab reporting and sending patient result text was collected.

Results 8 services took part. 0.1\% HIV positivity rate. $70 \%$ overall had documented reason for HIV test decline. 13\% were coded as declined with no documented offer. Percentage of people with needs relating to STI's who had an HIV test at first attendance $97 \%$ offered (achieved in $84 \%$, range 59 to $100 \%$ ), $80 \%$ uptake (achieved in $70 \%$, range 47 to $87 \%$ ). $3 / 8$ of services met both standards for turn-around times. Overall $92 \%$ of services received report from laboratory within 5 working days, range 1 to 20 days (standard 97\%) and 90\% of patients received their result within 10 working days, range 3 to 30 days (standard 95\%).
Discussion Not all patients appropriate to be tested were offered HIV test, training as to when HIV testing is not appropriate in Sexual health was recommended. Patients in a long term relationship were most likely not to be offered screening, regardless of previous screening history. There was a large variation between processing times in both laboratories and sexual health services. Good practices for those meeting standards were shared with the network.

\section{P054 PELVIC INFLAMMATORY DISEASE (PID) - IS TELEPHONE FOLLOW-UP FEASIBLE, SAFE AND EFFECTIVE?}

Myat Lwin* ${ }^{*}$ Sophie Forsyth, Jessica Daniel, Laura Hill. The Great Western Hospital, Swindon, UK

\subsection{6/sextrans-2016-052718.108}

Background BASHH guidelines recommend follow up after PID treatment. A previous clinic audit highlighted high DNA rates for such appointments. In October 2013 we introduced a telephone follow-up protocol for PID to reduce unattended appointments without compromising patient safety and satisfaction. Patients diagnosed with PID were referred to the Health Advisor (HA) at the first consultation to commence the Partner Notification (PN) process. HA's then conducted a telephone follow-up appointment 2 weeks later to ensure treatment compliance and review symptoms.

Aim To audit the performance of the new PID telephone followup protocol and estimate number of appointments saved.

Method A 3 months retrospective electronic case note and PN record review of female patients diagnosed with $\mathrm{C} 5 \mathrm{~A}$ attending between $1 / 7 / 14$ and 30/9/14.

Results 59 eligible case notes reviewed. Mean age $=25.8$ years. $66 \%(39 / 59)$ patients received telephone follow-up. 71\% (28/39) patients contacted on first attempt and all were happy to be telephoned. As per PID protocol 23\% (14/59) patients with positive Chlamydia, gonorrhoea test or IUD in situ were advised to attend for doctor review. Of these 36\% DNA'd their clinic follow up appointment. PN rates $0.8 \%$.

Discussion PID follow up performed by HA telephone consultation is acceptable to patients and HCP's. We saved 39 doctors appointments over 3 months and there was no impact on PN rates or patient safety. Since this audit we now include patients with Chlamydia and IUD's in the telephone follow-up protocol, and men with Epididymo- orchitis. We estimate we could save 280 follow-up appointments a year.

\section{P055 IMPROVING CLINICAL STANDARDS IN GU MEDICINE: A RETROSPECTIVE AUDIT OF NEISSERIA GONORRHOEA 2007 - 2015}

Sris Allan. Coventry \& Warwickshire Partnership NHS Trust, Coventry, UK

\subsection{6/sextrans-2016-052718.109}

Background/introduction This was a retrospective analysis of clinic performance in the management and treatment of Neisseria gonorrhoeae (GC) according to the current British Association of Sexual Health and HIV (BASHH) guidelines.

Methods All cases of GC diagnosed in our clinic between 1st January and 30th June 2015 were identified. The case notes were reviewed and assessed against current BASHH criteria. This was compared to data collected at the same clinic for the 